\title{
Efecto de la Aplicación de Láser de Baja Potencia Sobre la Mucosa Oral Lesionada
}

\author{
Effect of the Low-power Laser on the Oral Mucous Injured \\ "Iván Claudio Suazo Galdames; *** María Constanza Lara Sepúlveda; \\ "Mario Gonzalo Cantín López \& **Daniela Alejandra Zavando Matamala
}

SUAZO, G. I. C.; LARA, S. M. C.; CANTÍN, L. M. G. \& ZAVANDO, M. D. A. Efecto de la aplicación de láser de baja potencia sobre la mucosa oral lesionada. Int. J. Morphol., 25(3):523-528, 2007.

RESUMEN: La utilización del láser ha tenido una constante evolución y desarrollo. Se ha comprobado en numerosos estudios los efectos bioestimulantes que la irradiación láser de baja potencia produce al interactuar con los tejidos y células. Este estudio pretende determinar la existencia de diferencias morfológicas en la mucosa oral lesionada, posterior a la irradiación con láser de baja potencia, a través de cambios en la densidad vascular del plexo subpapilar de la mucosa oral.

Se utilizaron 15 ratas Sprague Dawley, las cuales se dividieron en tres grupos. A la totalidad de los animales se les lesionó la mucosa oral, en la zona media del frenillo labial inferior. El grupo I se tomó como control; al grupo II se le aplicó láser de baja potencia sobre la mucosa oral inmediatamente realizada la lesión, luego, a las 24 y 48 horas, en una dosis de 6 Joule/ $\mathrm{cm}^{2}$ por aplicación; al grupo III se le aplicó láser de baja potencia en una dosis única de $18 \mathrm{Joule} / \mathrm{cm}^{2}$ inmediatamente efectuada la lesión. La unidad de muestra y análisis fueron los cortes histológicos obtenidos de la sección de la mucosa oral lesionada.

Los resultados muestran una mayor densidad vascular en el grupo II con respecto a los grupos I y III, con una densidad promedio de 4,07 vasos sanguíneos $/ 25 \mathrm{~mm}^{2}$, mientras el grupo I de 2,47 vasos sanguíneos $/ 25 \mathrm{~mm}^{2}$ y el grupo III de 1,87 vasos sanguíneos/25mm². No se observó diferencia entre las densidades obtenidas para los grupos I y III. Estos resultados indican que la aplicación de láser de baja potencia en dosis baja produce un aumento en la densidad vascular en la mucosa oral lesionada.

PALABRAS CLAVE: Laserterapia de baja potencia; Láser bioestimulación; Mucosa oral.

\section{INTRODUCCIÓN}

Se entiende por regeneración, a la "sustitución de tejidos dañados o muertos por otros nuevos con la misma función”. El proceso de cicatrización es la sustitución de las células lesionadas por tejido conectivo originado del tejido de granulación.

La aplicación de radiación láser en dosis baja determina un incremento del proceso curativo en general, su efecto se basa en la multiplicación celular, la formación de fibras colágenas y elásticas, la regeneración de vasos, la cicatrización de tejido óseo y la reepitelialización del tejido dañado (Garrigo \& Valiente, 1996; Shamir et al., 1995).
Se deben distinguir dos grandes grupos de láser, los de alta potencia o quirúrgicos y los de baja potencia, también denominados terapéuticos. Los primeros tienen un efecto térmico, ya que son capaces de concentrar una gran cantidad de energía en un espacio muy reducido y ello se demuestra por su capacidad de corte, coagulación y vaporización. Por otro lado, el láser de baja potencia carece de este efecto térmico, ya que la potencia que utiliza es menor y la superficie de acción mayor, por lo que el calor se dispersa, produciendo además, un efecto bioestimulante celular. $\mathrm{Su}$ aplicación fundamental es acelerar la regeneración tisular y la cicatrización de las heridas disminuyendo la inflamación y el dolor (Oltra-Arimon et al., 2004).

\footnotetext{
* Unidad de Anatomía Normal, Facultad de Ciencias de la Salud, Universidad de Talca, Chile.

** Departamento de Estomatología, Facultad de Ciencias de la Salud, Universidad de Talca, Chile.

${ }^{* * *}$ Cirujano Dentista, práctica privada.
} 
El uso de láser de baja potencia se asocia al incremento de la microirrigación, mediada por fenómenos de angiogénesis, Paschoal et al. (2006) observaron un incremento de la irrigación de la porción distal de un colgajo irradiado con láser de baja potencia, mientras Souza et al. (2003) analizaron la presencia vasos sanguíneos neoformados y una alta proliferación celular en ratas irradiadas con láser de baja potencia.

Schaffer et al. (2000) mediante resonancia magnética, notaron un aumento significativo de flujo sanguíneo de la microcirculación del pié, cuando esta región fue irradiada con láser de baja potencia, sin haber alteración de la temperatura local, que fue avalada a través de dispositivos termosensibles en la región.

Moreira et al. (2006), tras un estudio realizado en ratas, sugieren que la terapia láser de baja potencia es eficaz en la cicatrización mostrando efectos positivos acelerando la proliferación celular, aumentando la vascularización local y formando un tejido de granulación más organizado.

Basado en estos antecedentes, en este trabajo se pretende cuantificar los cambios en la densidad vascular del plexo subpapilar de la mucosa oral lesionada, producidos por la aplicación de radiación láser de baja potencia.

\section{MATERIAL Y MÉTODO}

Se utilizaron 15 ratas Sprague Dawley, adultas, machos, de entre 250 y $300 \mathrm{~g}$ de peso. Éstas fueron adquiridas en el bioterio ANEXPA de la ciudad de Talca. Durante todo el periodo experimental, fueron alojadas en jaulas de uso habitual, al interior del laboratorio de anatomía de la Universidad de Talca, mantenidas con ciclos de $12-12 \mathrm{~h}$ luzoscuridad, con calefacción y con una dieta estándar de alimentos sólidos (pellet) y agua potable ad libitum. Este estudio se desarrolló en los laboratorios de Anatomía, Histopatología y Cultivo Celular de la Universidad de Talca, Chile.

Las 15 ratas fueron sometidas a anestesia general inhalada, utilizando éter etílico en una cámara de anestesia. Cuando en la rata se lograba una respiración toracoabdominal y ausencia de movimiento de los bigotes, se consideró que ésta se encontraba bajo anestesia profunda.

Una vez inducida la anestesia, a la totalidad de los animales se les lesionó la mucosa oral, mediante una incisión de $1,5 \mathrm{~mm}$, en la parte media del frenillo labial infe- rior, realizando una lesión de espesor total en la mucosa, con una hoja de bisturí $\mathrm{N}^{\circ} 11$ (Fig.1).

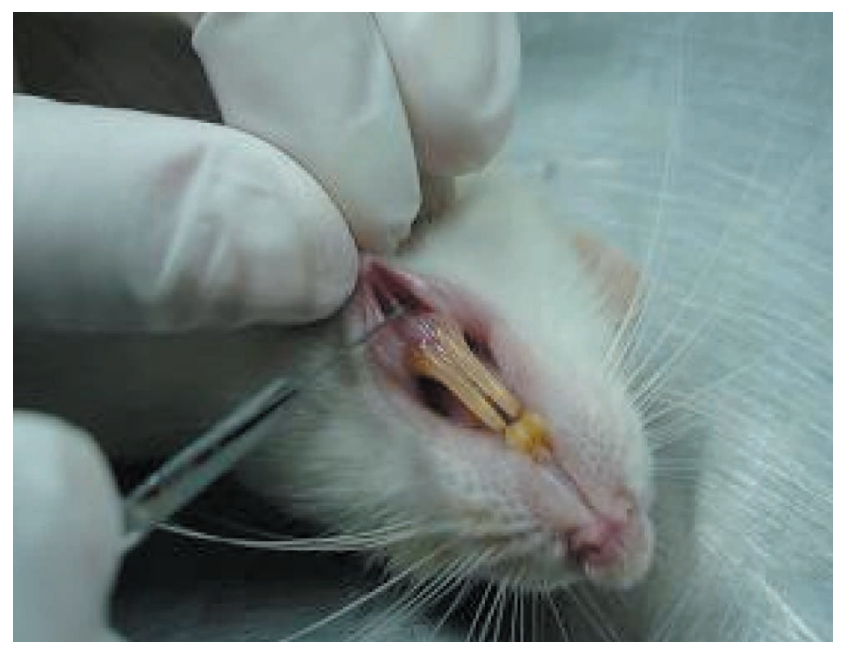

Fig. 1. Lesión del frenillo labial inferior.

Los ejemplares fueron divididos en 3 grupos: grupo I, de 5 ratas, como control; grupo II, de 5 animales, se le aplicó láser de baja potencia sobre la mucosa oral, inmediatamente después de realizada la lesión, luego, a las 24 y 48 horas en una dosis de $6 \mathrm{Joule} / \mathrm{cm}^{2}$ por aplicación; al grupo III, de 5 ratas, se le aplicó láser de baja potencia sobre la mucosa oral lesionada en una dosis única de 18 Joule $/ \mathrm{cm}^{2}$, inmediatamente después de efectuada la lesión. El equipo láser de baja potencia, Brightness - Kondortech ${ }^{\circledR}$ utiliza una fibra óptica de $2 \mathrm{~mm}$ de diámetro que conducía el rayo exactamente sobre el sitio lesionado, usando como referencia apoyar la fibra óptica en la zona cervical de los incisivos centrales inferiores (Fig. 2).

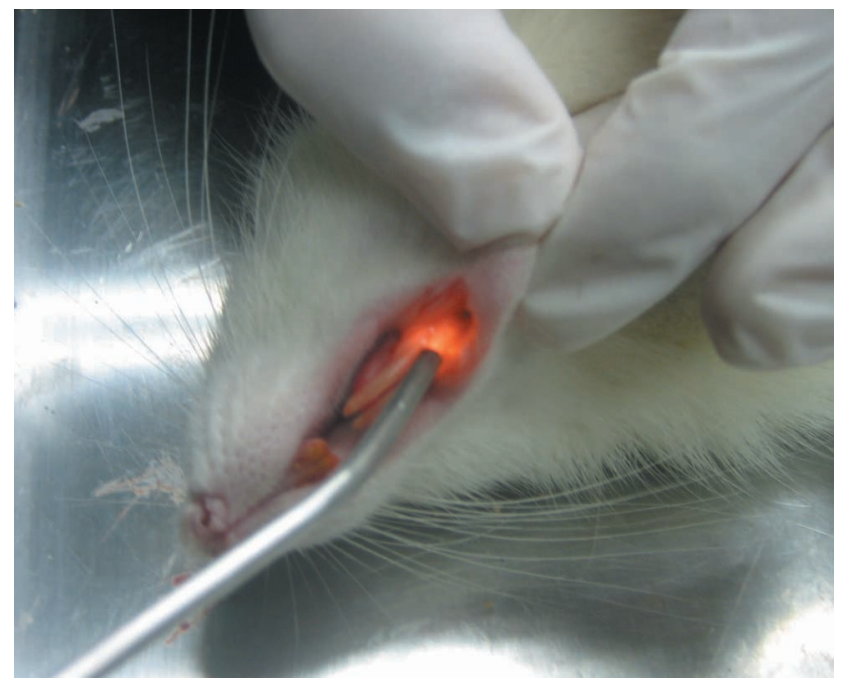

Fig. 2. Aplicación del láser dobre la mucosa lesionada. 
Todo el material utilizado en esta fase experimental se encontraba previamente esterilizado en autoclave Almed ( ) (modelo H.G.R.B.X.75).

A los 10 días de realizada la lesión, las ratas fueron nuevamente anestesiadas con éter etílico y se procedió a realizar la biopsia excisional estandarizada de la zona utilizando un bisturí circular de $4 \mathrm{~mm}$ de diámetro montado en un motor eléctrico Profile Dentsply®.

Las piezas de tejido se almacenaron en frascos individuales rotulados y fijadas en formalina al $10 \%$, para luego ser enviadas al Laboratorio de Histopatología de la Universidad de Talca, para ser procesadas para tinción de hematoxilina-eosina. Las piezas incluidas en parafina fueron sometidas a cortes seriados en sentido longitudinal, de $5 \mu \mathrm{m}$, con un micrótomo Shandon AS 325®.

El total de la muestra para este estudio fue de 15 ratas, las que se dividieron en tres grupos de 5 ratas, de cada una se analizaron 3 cortes histológicos por lo que cada grupo tiene un total de 15 muestras.

Para el análisis cuantitativo se utilizaron 3 placas histológicas obtenidas de la región central de la biopsia de cada espécimen, las cuales fueron observadas con microscopio invertido trinocular Nikon Eclipse TS100, fotografiando el plexo subpapilar con una cámara fotográfica Nikon Coolpix 4300.

Se obtuvieron 15 imágenes por cada grupo, las cuales fueron procesadas con el Software Macromedia Freehand.MXa. Para evaluar la densidad vascular por grupo, en cada muestra se realizó el conteo de número de vasos sanguíneos del plexo subpapilar en $25 \mathrm{~mm}^{2}$

La información recopilada, puesta en fichas de recolección de datos, se agrupó de acuerdo a los periodos de estudio y los datos fueron ordenados en el programa estadístico SPSS 10.0 del sistema operativo Windows XP. Se utilizó la prueba no paramétrica de Mann-Whitney.

\section{RESULTADOS}

En el grupo I el promedio del $\mathrm{N}^{\mathrm{o}}$ de vasos sanguíneos del plexo subpapilar (densidad vascular) fue de 2,47 (DS 2,72); en el grupo II el promedio fue de 4,07 (DS 1,79); en el grupo III el promedio fue de 1,87 (DS 0,92), estos resultados son presentados en la Tabla I.

En la Fig. 3 se compara la densidad vascular para los grupos I, II y III. Se observa una mayor densidad vascular del grupo II con respecto a los grupos I y III, al presentar una densidad promedio de 4,07 correspondiente, aproximadamente, al doble de la densidad promedio para el grupo I $(2,47)$ y grupo III $(1,87)$; no existiendo una marcada diferencia entre las densidades obtenidas para los grupos I y III.

Tabla I. Densidad vascular en el plexo subpapilar en los grupos I, II y III.

\begin{tabular}{|c|c|c|c|c|}
\hline \multirow[b]{2}{*}{ Grupo } & \multicolumn{4}{|c|}{ Densidad vascular } \\
\hline & $\begin{array}{c}\text { Valor } \\
\text { promedio }\end{array}$ & $\begin{array}{l}\text { Valor } \\
\text { mínimo }\end{array}$ & $\begin{array}{l}\text { Valor } \\
\text { máximo }\end{array}$ & $\begin{array}{c}\text { Desviación } \\
\text { estándar }\end{array}$ \\
\hline $\mathrm{I}$ & 2,47 & 0 & 9 & 2,72 \\
\hline II & 4,07 & 1 & 8 & 1,79 \\
\hline III & 1,87 & 0 & 3 & 0,92 \\
\hline
\end{tabular}

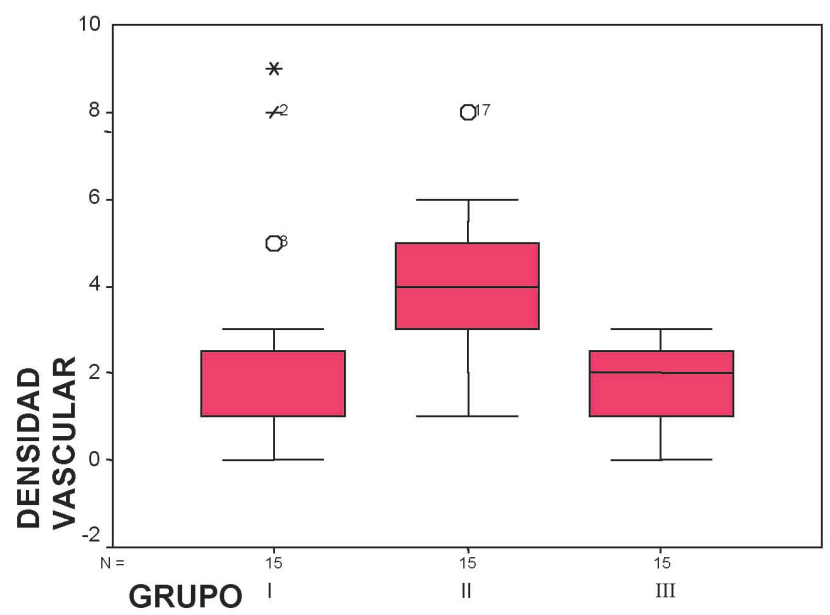

Fig. 3. Densidad vascular por grupo.

En la Tabla II se expresan los rangos promedios para los grupos I y II, según la densidad vascular. En el análisis total se puede observar que el grupo II presentó un aumento en la densidad vascular. Esta diferencia resultó estadísticamente significativa entre ambos grupos $(\mathrm{p}<0,05)$.

Tabla II. Datos estadísticos de contraste, prueba de Mann-Whitney, entre grupos I y II.

\begin{tabular}{cccc}
\hline & \multicolumn{3}{c}{ Densidad vascular } \\
Grupo & $\mathrm{n}$ & Rango promedio & $\mathrm{p}$ \\
\hline I & 15 & 11,50 & $0,011^{*}$ \\
II & 15 & 19,50 & \\
Total & 30 & & \\
\hline *Significancia estadística & &
\end{tabular}

*Significancia estadística

En la Tabla III se expresan los rangos promedios para los grupos I y III según la densidad vascular. Al comparar los valores de densidad vascular, el rango promedio para el grupo I fue de 14,57 en comparación con el grupo III, cuyo 
rango promedio fue de 16,43 , esto indica una menor densidad vascular a nivel del grupo I; no obstante, no se observaron diferencias estadísticamente significativa entre ambos grupos $(\mathrm{p}<0,05)$.

Tabla III. Datos estadísticos de contraste, prueba de Mann-Whitney, entre grupos I y III.

\begin{tabular}{cccc}
\hline \multicolumn{4}{c}{ Densidad vasc ular } \\
Grupo & $\mathrm{n}$ & Rango promedio & $\mathrm{p}$ \\
\hline I & 15 & 14,57 & $0,54 *$ \\
III & 15 & 16,43 & \\
Total & 30 & & \\
\hline *Significancia estadística & &
\end{tabular}

En la Tabla IV se expresan los rangos promedios para los grupos II y III, según la densidad vascular. En el análisis total se puede observar que el grupo que presentó un aumento en la densidad vascular fue el grupo II. Esta diferencia fue estadísticamente significativa entre ambos grupos $(\mathrm{p}<0,05)$.

Tabla IV. Datos estadísticos de contraste, prueba de Mann-Whitney, entre grupos II y III.

\begin{tabular}{cccc}
\hline Grupo & \multicolumn{2}{c}{ Densidad vascular } & \\
II & 15 & Rango promedio & $\mathrm{p}$ \\
\hline III & 15 & 21,00 & $0,00^{*}$ \\
Total & 30 & 10,00 & \\
\hline
\end{tabular}

*Significancia estadística

\section{DISCUSIÓN}

El efecto de láser de baja potencia sobre distintos tejidos orales ha sido estudiado por numerosos autores (Greathouse et al., 1985; Almeida, 2004; Oltra-Arimon et al.; Garrigo \&Valiente), reconociéndose un efecto benéfico en la cicatrización de heridas de la mucosa oral y en otros tejidos

Se han propuestos diferentes mecanismos de acción del láser de baja potencia, favoreciendo el proceso de reparación, su acción se basa en la multiplicación celular, la formación de fibras colágenas y elásticas, la reepitelialización del tejido dañado; sobre las células del endotelio vascular incrementa la actividad mitótica y se producen aceleradamente yemas o brotes de los vasos existentes para la neoformación de microvasos (Garrigo \& Valiente).

En el presente estudio se analizaron los cambios en la densidad vascular asociados a la aplicación de lá- ser de baja potencia, en mucosa que había sido previamente lesionada. Mediante el análisis histológico de las muestras obtenidas se observó que, si bien hubo cambios morfológicos en los tres grupos en estudio, estos cambios no afectaron a los tres grupos por igual. Al comparar el grupo control (grupo I) con el grupo irradiado en dosis baja (6 Joules $/ \mathrm{cm}^{2}$ ) aplicada en tres ocasiones (grupo II) se observó un mayor número de vasos sanguíneos en el plexo subpapilar por unidad de superficie; esta diferencia resultó estadísticamente significativa $(\mathrm{p}<0,005)$. Esto en general, coincide con lo manifestado en la literatura, en relación al efecto del láser de baja potencia en los cambios microvasculares que experimenta el tejido conectivo, afirmando que existe una proliferación en la microrred de vasos sanguíneos (Moreira et al.; Boulton \& Marsal, 1986; Curri \& Campi, 1986; Kameya, 1995; Garrigo, \& Valiente).

Por otro lado, al analizar la densidad vascular del plexo subpapilar en el grupo control (grupo I) y compararla con la presentada por el grupo irradiado en dosis alta (18 joules/ $\mathrm{cm}^{2}$ ) aplicada en una sola ocasión, inmediatamente post-lesión (grupo III), se observaron resultados promedio levemente superiores en el grupo I; no obstante, esta diferencia no resultó estadísticamente significativa ( $p>0,005)$. Estos resultados concuerdan con los de algunos autores quienes manifiestan que la aplicación de láser de baja potencia en dosis elevada, no favorece las respuestas reparativas tisulares (Greathouse et al.; Garrigo \&Valiente; Braverman et al.,1989; Skinner et al., 1996 ).

Al analizar la densidad del plexo subpapilar en el grupo irradiado en dosis baja (6 joules/ $\mathrm{cm}^{2}$ ) aplicada en tres ocasiones (grupo II) y compararlo con el grupo III que recibió una dosis única alta (18 joules $\left./ \mathrm{cm}^{2}\right)$, se observó una mayor cantidad de vasos sanguíneos por unidad de superficie en el grupo II, lo cual resultó estadísticamente significativo ( $\mathrm{p}<0,005)$, en concordancia con lo expresado por Garrigo \& Valiente, quienes describieron que el uso de altas dosis de láser de baja potencia produce una inhibición de la multiplicación celular y con ello una disminución de las respuestas tisulares asociadas a la reparación, entre ellas la proliferación microvascular.

El análisis de estos resultados nos lleva a pensar que, en determinadas condiciones, el uso de láser de baja potencia sobre los tejidos, favorece la respuesta reparativa, mediante el aumento de la vascularización, lo que se correlaciona con lo comunicado por Miro \& Caupe (1984), quienes demostraron que el láser produce un aumento en la vascularización que repercute sobre el número de mitosis celulares. 
SUAZO, G. I. C.; LARA, S. M. C.; CANTín, L. M. G. \& ZAVANDO, M. D. A. Effect of the low-power laser on the oral mucous injured. Int. J. Morphol., 25(3):523-528, 2007.

SUMMARY: The laser use has had a constant evolution and development; the bioestimulantion effects have been verified in numerous studies that the irradiation laser of fall promotes produces on having interacted with cells and tissues This study tries to determine the morphologic differences in the oral mucous injured, after the application of low-level laser, across changes in the vascular density of the subpapilar plexus of the mucous oral.

Fifteen Sprague Dawley mice were used, which formed three groups, in the total number of animals the oral mucous was injured, in the average zone of the labial low frenum. Group I was taken as a control; in group II a low-level laser was applied on the mucous immediately the oral injury, then, at 48 after 24 hours in a dose of 6 Joule $/ \mathrm{cm}^{2}$ for application; Group III a low-level laser in a single dose of $18 \mathrm{Joule} / \mathrm{cm}^{2}$ was applied immediately the oral injury. The unit of sample and analysis they were the histological cuts obtained of the section of the oral mucous injured.

The results show a major vascular density in the group the II with regard to the group I and the III, with a density I mediate of 4,07 vassels $/ 25 \mathrm{~mm}^{2}$, while the group I of 2,47 vassels $/ 25 \mathrm{~mm}^{2}$ and group II of 1,87 vassels $/ 25 \mathrm{~mm}^{2}$. No find differences between groups I and III. These results indicate that the application of low-level laser in low dose originate an increase in the vascular density in the oral mucous injured.

KEY WORDS: Low level laser therapy; Laser biostimulation; Oral mucous.

\section{REFERENCIAS BIBLIOGRÁFICAS}

Almeida, L. Laserterapia en odontología. Biodonto., 1:9-87, 2004.

Boulton, H. \& Marsal, J. Laser stimulation of human fibroblast proliferation and attachment in vitro. Laser lefe Sci., 1:125-34, 1986.

Braverman, B.; McCarthy, R.; Ivankovich, A.; Forde, D.; Overfield, M. \& Bapna, M. Effect of helium-neon and infrared laser irradiation on wound healing in rabbits. Lasers Surg. Med., 9:50-8, 1989.

Curri, S. \& Campi, E. Tessuto connecttivo e microvascolarizzazione. En: Franco Bistolfy. Campi magnetici in medicina, Torino, 1986. pp. 57-441.

Garrigo, M. \& Valiente, C. Efectos biológicos de la radiación láser de baja potencia en la reparación hística. Rev. Cubana Estomatología, 33(2):60-3, 1996.

Greathouse, D.; Currier, D. \& Gilmore, R. Effects of clinical infrared laser on superficial radial nerve conduction. Phys. Ther., 65:84-5, 1985.

Kameya, T. Effect of different wavelengths of low level laser therapy on wound healding in mice. Laser Ther., 7:336, 1995.

Miro, H. \& Caupe, C. Estudio capilaroscópico de la acción del láser As Ga. Invest. Clin. Láser, 2:9-13, 1984.
Moreira, A.; Guerra de Oliveira, R.; Farias, R.; Ferreira, L. \& Monteiro, F. Modulação da proliferação fibroblástica e da resposta inflamatória pela terapia a laser de baixa intensidade no processo de reparo tecidual. An. Bras. Dermatol., 81(2), 2006.

Oltra-Arimon, D.; España-Tost, A.; Berini-Aytés, L. \& GayEscoda, C. Aplicaciones del láser de baja potencia en Odontología. RCOE, 9(5):517-24, 2004.

Paschoal, R.; Eloin, R.; Hochman, B.; Pinfildi, C. \& Masako, L. Experimental model for low level laser therapy on ischemic random skin flap in rats. Acta Cir. Bras., 21(4): 2006.

Schaffer, M.; Bonel, H.; Sroka, R.; Schaffer, P.; Busch, M.; Sittek, H.; Reiser, M. \& Duhmke, E. Magnetic resonance imaging (MRI) controlled outcome of side effects caused by ionizing radiation, treated with $780 \mathrm{~nm}$-diode laser, preliminary results. J. Photochem. Photobiol. B., 59(13):1-8, 2000.

Shamir, M.; Rochkind, S.; Sandbank, J. \& Alon, M. Doubleblind study evaluating regeneration of the rat transected sciatic nerve after suturing and postoperative low-power laser treatment. Eur. J. Oral Sci., 103(5):299-305, 1995.

Skinner, S.; Gage, J.; Wilce, P.; Shaw, R. Preliminary study of the effects of laser radiation on collagen metabolism in cell. Culture Australian Dental J., 41:188-92, 1996. 
Souza, L.; Peixoto, A.; Almeida, S. \& Araújo, Z. A influência da terapia a laser de baixa densidade de energia na biomodulação das fibras colágenas e elásticas. Pesquiza Odontol. Bras., 17(4): 2003.
Dirección para correspondencia:

Prof. Dr. Iván Suazo Galdames

Unidad de Anatomía Normal

Universidad de Talca Avenida

Lircay s/n oficina $\mathrm{N}^{\circ} 104$

Talca - CHILE

Fono 56-71-201682

Email: isuazo@utalca.cl

Recibido : 14-03-2007

Aceptado: 20-04-2007 